\title{
Chairman-Lecture
}

\section{The essential values of HBP surgery in my life}

\author{
Hee Chul YU
}

Department of Surgery, Jeonbuk National University Medical School, Jeonju, Korea

Lecture: Essential values are the fundamental beliefs of a person or organization. It guiding principles dictate behavior and can help people understand the difference between right and wrong. Positive values help person to determine if they are on the right path and fulfilling their goals by creating an unwavering guide for living a good life and negative core values may be driven by self-interest or greed. There are many different examples of essential values, depending upon the context. Everyone one has their own valuable things which are important in the way of live and work.

In the HBP surgery field, I would like to prioritize 8 essential values such as knowledgeable, passion, collaborative, innovative, progressive, openness, excellence, and leadership. I'd like to talk about some of them in this talk that dictate how life should be lived based on my experience. 